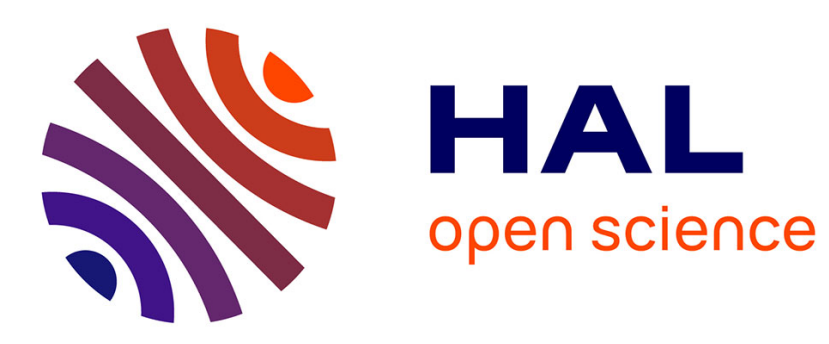

\title{
Effets du remodelage parcellaire sur la fixation des phosphates en divers types de sols des Antilles
}

Théodore Chevignard, Jean-Claude Fardeau, Sylvie Doulbeau-Piasco, Christian Feller, Jean-François Turenne, Michel Vallerie

\section{- To cite this version:}

Théodore Chevignard, Jean-Claude Fardeau, Sylvie Doulbeau-Piasco, Christian Feller, Jean-François Turenne, et al.. Effets du remodelage parcellaire sur la fixation des phosphates en divers types de sols des Antilles. Agronomie, 1986, 6 (2), pp.149-156. hal-00884859

\section{HAL Id: hal-00884859 \\ https://hal.science/hal-00884859}

Submitted on 1 Jan 1986

HAL is a multi-disciplinary open access archive for the deposit and dissemination of scientific research documents, whether they are published or not. The documents may come from teaching and research institutions in France or abroad, or from public or private research centers.
L'archive ouverte pluridisciplinaire HAL, est destinée au dépôt et à la diffusion de documents scientifiques de niveau recherche, publiés ou non, émanant des établissements d'enseignement et de recherche français ou étrangers, des laboratoires publics ou privés. 


\title{
Effets du remodelage parcellaire sur la fixation des phosphates en divers types de sols des Antil-
} les

\author{
Théodore CHEVIGNARD, Jean-Claude FARDEAU $\left({ }^{*}\right)$, Sylvie DOULBEAU-PIASCO $\left({ }^{* *}\right)$, Christian FEL- \\ LER, Jean-François TURENNE \& Michel VALLERIE $\left({ }^{* *}\right)$
}

O.R.S.T.O.M., B.P. 81, F 97200 Fort de France Cedex, Martinique

(*) C.E.N. Cadarache, D.B., S.R.A., B.P. I, F 13115 Saint-Paul-lez-Durance

(**) Antenne O.R.S.T.O.M., C.E.N. Cadarache, B.P. I, F 13115 Saint-Paul-lez-Durance

Mots clés additionnels : ${ }^{32} P$, cinétique, dilution isotopique, sols ferrallitiques, andosols.

\section{Effect of hill shaping on phosphate fixation in different West Indian soil types}

Levelling of the soil for mechanical agriculture brings to the top soil a layer poor or very poor in organic matter content. Experiments were conducted by isotope methods to analyse the phosphate fertility of some typical West Indian soils. It was concluded that all the soils were not only poor in available soil phosphorus but also had a very high fixing capacity for phosphate ions, and that the fixing capacity of top soil for phosphorus was lower than for the subsoil. This observation explains the increased fixing capacity of the soils modified by hill shaping. A positive effect of organic matter appears from the comparison of different soil types. The more water-soluble $\mathrm{P}$ fertilizers, such as diammonium phosphate, were fixed very quickly : $96 \%$ in $24 \mathrm{~h}$. For this reason and due to soil acidity, the use of soft rock-phosphates remains the best choice for phosphate fertilization.

Additional key words : ${ }^{32} P$, isotope kinetics, ferrallitic soils, andosoils.

\section{INTRODUCTION}

Aux Antilles françaises, des opérations de nivellement des terrains, appelées « remodelage des sols", ont été menées de 1970 à 1975 dans le but d'accroître la mécanisation des cultures. Des épaisseurs de sols variant de 0,5 à $6,0 \mathrm{~m}$ ont été ainsi décapées ou remaniées, ce qui conduit le plus souvent à la disparition de l'horizon humifère $A$ et à la mise en surface d'horizons $\mathrm{B}$ ou $\mathrm{C}$ très pauvres en matière organique. En
Martinique, environ 70 p. 100 de surfaces cultivées en canne à sucre ont été concernées par ces opérations qui ont représenté un investissement de 10 millions de francs.

Après 15 ans de culture de canne à sucre, le stock organique des différents types de sols ainsi remodelés (sols bruns, ferrallitiques et andiques) n'atteint que 40 p. 100 de celui des sols correspondants non remodelés, et la production végétale reste faible même en présence d'une forte fumure minérale (CHEvigNARD, 
1982). Par exemple, dans un sol qui sera analysé au cours de cette étude, le rendement est de $92 \mathrm{t}$. ha ${ }^{-1}$ sur le sol témoin et de $45{\mathrm{t} . \mathrm{ha}^{-1}}^{-1}$ sur le sol remodelé.

Nous analysons ici les conséquences de ces opérations de nivellement sur la fertilité phosphorique dans différentes situations, et, en particulier, sur le pouvoir fixateur des sols à l'égard du phosphore avec l'objectif de proposer une fertilisation phosphatée adaptée à ces types de sols.

Partant de recherches originales sur la mobilité des ions phosphatés dans les sols (BARBIER \& TYSZKIEWISZ, 1952 ; BARBIER \& TROCMÉ, 1964), des études sont entreprises depuis quelques années (FARDEAU, 1981) pour caractériser le pool assimilable des ions $\mathrm{PO}_{4}^{3-}$ dans le système sol-plante, non seulement par la quantité des espèces en présence (ions libres, mobiles ou fixés) mais aussi par leur cinétique d'échange : on utilise à cette fin la méthode des cinétiques de dilution isotopique dont l'objectif est de quantifier des paramètres caractérisant les échanges d'ions $\mathrm{PO}_{4}^{3-}$ entre le pool des ions libres et l'ensemble des ions mobiles. C'est cette technique qui est appliquée ici sur des échantillons témoins ou enrichis par des apports d'engrais phosphatés.

Diverses méthodes ont été proposées pour quantifier le pouvoir fixateur des sols vis-à-vis des phosphates, celui-ci étant généralement déterminé à partir d'ajouts croissants de phosphate. Ainsi, par exemple, à partir du concept de dose isodyname (DEMOLON et al., 1953), il a été défini comme la quantité de phosphore à apporter à un sol pour atteindre la concentration en solution de $2 \mathrm{mg} \mathrm{P} . \mathrm{l}^{-1}$ pour un rapport solsolution 1/4 (GACHON, 1969). De même, parce que, pour certains, les cultures atteindraient 95 p. 100 de leur rendement maximum si la solution du sol avait une concentration permanente de 0,2 p.p.m. P, on détermine parfois la quantité de phosphore nécessaire pour atteindre cette concentration (FOX \& KAMPRATH, 1970). Dans ce cas, les quantités à apporter sont souvent très élevées au regard des doses d'engrais possibles et SHELDON (1982) signale que l'obtention de telles concentrations sur l'ensemble des sols agricoles nécessiterait d'utiliser immédiatement la moitié des réserves mondiales en phosphates. C'est pourquoi il a semblé préférable de chercher à caractériser le pouvoir fixateur du sol en l'état, c'est-à-dire sans faire appel à un apport de phosphate. Tel est l'objet d'un des paramètres obtenu au cours des déterminations des cinétiques de dilution isotopique.

\section{MATÉRIEL ET MÉTHODES}

\section{A. Technique des cinétiques de dilution isotopique}

\section{Réalisation expérimentale}

Dans un mélange sol-eau de rapport $1 / 10$, agité une nuit pour obtenir un état d'équilibre, on injecte de 0,1 à $1 \mathrm{M} \mathrm{Bq}$ de ${ }^{32} \mathrm{P}$ sous forme de ${ }^{32} \mathrm{PO}_{4}^{3-}$ sans entrâ̂neur. On prélève régulièrement à l'aide d'une seringue de 5 à $8 \mathrm{ml}$ de suspension dans le mélange agité à 200 tours/mn environ, et l'on filtre immédiatement la solution sur des membranes type « millipore » à pores de $0,2 \mu \mathrm{m}$; les prélèvements ont lieu à 1,10 et $100 \mathrm{mn}$ après l'instant d'injection du traceur. La radioactivité des solutions filtrées est déterminée par scintillation liquide. A l'issue du dernier prélèvement, on centrifuge le mélange pendant $40 \mathrm{mn}$ à $140000 \mathrm{~g}$ afin de déterminer par colorimétrie la quantité $\mathrm{M}_{1}$ d'ions $\mathrm{PO}_{4}^{3-}$ en solution (FARDEAU, 1981).

\section{Equation d'une cinétique de dilution isotopique}

Pour la majorité des sols, il existe une équation simplifiée (FARDEAU et al., 1985) susceptible de décrire la cinétique d'échange entre la fraction libre et la fraction retenue des ions phosphates mobiles.

Elle est de la forme :

$$
\frac{\frac{r}{R}}{M_{1}}=\frac{\frac{r_{1}}{R}}{M_{1}} \cdot t^{-n}
$$

La signification des symboles est la suivante :

- $\mathrm{R}$ est la radioactivité totale introduite dans le système ;

- $r$ est la radioactivité présente en solution sous forme d'ions ${ }^{32} \mathrm{PO}_{4}^{3-}$ à l'instant $t$, généralement exprimé en minutes ;

- $r_{1}$ est la radioactivité présente en solution après 1 minute d'échange ;

- $M_{1}$ est la quantité de phosphore présente en solution sous forme d'ions $\mathrm{PO}_{4}^{3-}$; elle est dosée par réduction du complexe phospho-mobybdique soit par du sulfate d'hydrazine à chaud (MARINI, 1965), soit par de l'acide ascorbique à froid (JOHN, 1970). Le choix de l'une ou l'autre méthode résulte de la présence des substances interférentes : silice et nitrates en particulier ;

- $n$ est un exposant compris entre 0 et 0,5 .

La caractérisation du phosphore mobile d'un sol repose donc sur 3 déterminations : $M_{1}$, n et $r_{1} / R$ qui permet de caractériser le pouvoir fixateur des sols pour le phosphore (FARDEAU \& JAPPE, 1982).

\section{B. Les sols étudiés}

Les échantillons de surface, $\mathrm{s}(0-15 \mathrm{~cm})$ et de profondeur, p $(30-50 \mathrm{~cm})$ proviennent de 3 types de sols développés sur matériaux volcaniques et bien représentés en Martinique (COLMET-DAAGE \& LAGACHE, 1965) :

- les sols ferrallitiques (ferrisols) à dominance de kaolinite dans la fraction argileuse,

- les sols brunifiés (sols brun-rouille) à dominance d'halloysite dans la fraction argileuse,

- les andosols à dominance d'allophanes dans la fraction argileuse.

Pour chaque type de sol, on distingue 3 traitements « végétation »:

- végétation arborée de plus de 20 ans appelé «Témoin Naturel » (TN),

- cultures de canne à sucre ou de banane de plus de 5 ans sur sols non remodelés appelé « Témoin Cultivé » (TC),

- cultures de canne à sucre ou de banane de plus de 5 ans sur sols remodelés appelé « Remodelé » (R). Le nombre d'années écoulées depuis l'action de remodelage est indiquée en indice (ex. : $R_{10}$ ). 
Les échantillons des traitements $\mathrm{R}$ sur ferrisols et andosols proviennent des horizons d'altération de ces sols tandis que ceux correspondant aux sols brun rouille sont issus d'horizons d'altération de ferrisols enterrés. Il ne nous a pas été possible de trouver une situation de remodelage sur matériau riche en halloysite (sol brun).

La minéralogie des argiles est étudiée par diffractométrie aux RX.

L'analyse mécanique est faite selon la Méthode Internationale avec détermination des fractions argiles A et limons fins LF $(0-2$ et $2-20 \mu \mathrm{m})$ par prélèvement à la pipette Robinson. Pour les échantillons à allophanes, la dispersion est assurée, si nécessaire, à $\mathrm{pH}$ variable, basique, acide et neutre (COLMET-DAAGE $e t$ al., 1972).

La capacité d'échange est déterminée à $\mathrm{pH} 7,0$ en analyse de routine par la méthode à l'acétate d'ammonium bien que, comme l'ont montré de nombreux auteurs (OUVRY, 1985), cette technique ne soit pas adaptée au cas des sols à charges variables, ce qui est en particulier le cas ici des andosols.

Les teneurs en $\mathrm{C}$ et $\mathrm{N}$ sont mesurées à l'aide d'un analyseur élémentaire Carlo Erba Mod-1106.

Le phosphore total est déterminé après une attaque nitrique, par la méthode de MISSON (phospho-vanadomolybdate) et le phosphore "assimilable ", extrait par $\mathrm{H}_{2} \mathrm{SO}_{4}, 0,02 \mathrm{~N}$ (AYRES \& HAGIHARA, 1961), est dosé par une méthode au bleu (MARINI, 1965).
Quelques caractéristiques des échantillons étudiés sont présentées dans les tableaux 1 et 2 .

Les teneurs en matière organique varient avec les traitements (TN $>\mathrm{TC}>\mathrm{R}$ ), les types de sols pour le traitement sous végétation naturelle (andosol $>$ ferrisol) et la profondeur de prélèvement $(s>p)$.

La minéralogie des argiles confirme, pour l'essentiel, la classification des sols : kaolinite pour les ferrisols, halloysite pour les sols brun rouille, allophane pour les andosols.

Seul le sol brun rouille $\mathrm{R}_{10}$ correspond à un horizon d'altération de ferrisol comme le montre la comparaison avec le ferrisol $R_{12}$.

\section{Les apports d'engrais phosphatés au laboratoire}

Le phosphore a été apporté sous forme de phosphate d'ammonium en raison de l'utilisation actuelle de cet engrais en Martinique sur canne à sucre.

Les doses retenues $\mathrm{P}_{0}, \mathrm{P}_{1}$ et $\mathrm{P}_{2}$, respectivement égales à 0,33 et $66 \mu \mathrm{g} \mathrm{P} \cdot \mathrm{g}^{-1}$ sol sont supérieures à celles appliquées au champ sur culture de canne (13 p.p.m. P) ; le fort pouvoir fixateur des sols étudiés justifie ce choix.

Pour améliorer la précision des apports, l'engrais a été apporté en solution, de préférence à la forme solide, $18 \mathrm{~h}$ avant le début des déterminations de cinétiques de dilution isotopique.

TABLEAU 1

Principales caractéristiques physico-chimiques des horizons de surface $(0-15 \mathrm{~cm})$. Main physical-chemical characteristics of the top soil $(0-15 \mathrm{~cm})$.

\begin{tabular}{|c|c|c|c|c|c|c|c|c|c|c|c|}
\hline \multirow{2}{*}{$\begin{array}{l}\text { Type de } \\
\text { sol }\end{array}$} & \multirow{2}{*}{$\begin{array}{l}\text { Culture } \\
\text { et site }\end{array}$} & \multirow{2}{*}{$\begin{array}{l}\text { Echan- } \\
\text { tillon }\end{array}$} & \multirow{2}{*}{$\begin{array}{l}\text { Minéralogie } \\
\text { (argiles) }\end{array}$} & \multicolumn{5}{|c|}{ Bases échangeables (meq. $100 \mathrm{~g}$ ) } & \multirow{2}{*}{$\begin{array}{c}\mathrm{Fe} \\
\text { (TAMM) } \\
\% \\
\% 0\end{array}$} & \multirow{2}{*}{$\mathrm{pH}$} & \multirow{2}{*}{$\begin{array}{l}\text { CEC } \\
\text { meq/ } \\
100 \mathrm{~g}\end{array}$} \\
\hline & & & & $\mathrm{K}$ & $\mathrm{Ca}^{++}$ & $\mathrm{Mg}^{++}$ & $\mathrm{S}$ & $\mathrm{Al}^{+++}$ & & & \\
\hline \multirow{4}{*}{ Ferrisol } & \multirow{4}{*}{$\begin{array}{c}\text { Canne } \\
\text { (le Gallion) }\end{array}$} & TNs & nd & & & & & 0,0 & 1 & 6,2 & 24 \\
\hline & & TCs & $\mathrm{FC} / \mathrm{M} / \mathrm{Go}, \mathrm{He}$ & 0,17 & 8 & 2,7 & 11,5 & 0,2 & 7,8 & 5,2 & 18,0 \\
\hline & & R10s & $\begin{array}{c}(+/+/ \mathrm{t}) \\
\mathrm{FC} / \mathrm{M} / \mathrm{Go}, \mathrm{He} \\
(+/+/ \mathrm{t})\end{array}$ & 0,36 & 7,9 & 7,8 & 16,7 & 0,1 & 1,8 & 5,2 & 25,5 \\
\hline & & $\mathrm{R} 12 \mathrm{~s}$ & $\begin{array}{c}\text { FCou MH/Go, He } \\
(++/ t)\end{array}$ & 0,24 & 4,8 & 3,7 & 10,5 & 0,1 & 1 & 5,6 & 18,0 \\
\hline \multirow[b]{3}{*}{$\begin{array}{l}\text { Sol brun } \\
\text { rouille }\end{array}$} & \multirow[b]{3}{*}{$\begin{array}{c}\text { Canne } \\
\text { (Ste Marie) }\end{array}$} & TNs & nd & & & & & 0 & \multirow{3}{*}{5,1} & 6,1 & 28 \\
\hline & & TCs & $\mathrm{H} / \mathrm{Cri}$ & 0,9 & 4,0 & 1,4 & 7,5 & 1,8 & & 4,8 & 23,5 \\
\hline & & R10s & $\begin{array}{c}(++/ t) \\
\text { FCou MH/M, Cri } \\
(++/ t)\end{array}$ & 0,2 & 2,7 & 1,9 & 5,0 & 0,2 & & 4,7 & 12,5 \\
\hline \multirow{6}{*}{ Andosol } & \multirow{3}{*}{$\begin{array}{c}\text { Canne } \\
\text { (St Etienne) }\end{array}$} & TNs & nd & & & & & 0,05 & 1 & 5,9 & 23,5 \\
\hline & & TCs & $\begin{array}{l}\text { All/MH, Gi } \\
(++/ t)\end{array}$ & 0,07 & 1,5 & 0,9 & 3,0 & 0,15 & 9,2 & 5,0 & 18,5 \\
\hline & & R8s & $\begin{array}{l}\text { All/Gi, Cri } \\
(++/ t)\end{array}$ & 0,08 & 3,7 & 1,2 & 5,8 & 0,02 & 5,6 & 5,6 & 22,5 \\
\hline & \multirow[b]{3}{*}{$\begin{array}{l}\text { Banane } \\
\text { (St Joseph) }\end{array}$} & TNs & nd & & & & & & 0,25 & 6,2 & 22,0 \\
\hline & & TCs & All/FC/Gi, Cri, He & & & & & & 1 & 5,7 & 22,0 \\
\hline & & $\mathrm{R} 7 \mathrm{~s}$ & $\begin{array}{c}\text { All } \\
(++)\end{array}$ & & & & & & / & 5,5 & 15,5 \\
\hline
\end{tabular}

Symboles : $\mathrm{TC}=$ Témoin cultivé (non remodelé), $\mathrm{R}=$ remodelé $(10)$ âge de remodelage, $\mathrm{TN}=$ témoin sous végétation naturelle.

Minéralogie : $\mathrm{FC}=$ kaolinite, $\mathrm{M}=$ montmorillonite, $\mathrm{Go}=$ goethite, $\mathrm{He}=$ hématite, $\mathrm{MH}=$ métahalloysite, $\mathrm{H}=$ halloysite, $\mathrm{Cri}=$ cristobalite, $\mathrm{All}=$ allophanes, $\mathrm{Gi}=$ gibbsite. 
TABLEAU 2

Teneurs en matière organique, phosphore et éléments fins $(A+L F=0-20 \mu \mathrm{m})$ des échantillons de surface $(0-15 \mathrm{~cm})$ et de profondeur $(30-50 \mathrm{~cm})$.

Organic matter, phosphorus, silt (A) and loam (LF) contents in 0-15 and 30-50 cm layers.

\begin{tabular}{|c|c|c|c|c|c|c|c|c|}
\hline \multirow{2}{*}{$\begin{array}{l}\text { Type de } \\
\text { sol }\end{array}$} & \multirow{2}{*}{$\begin{array}{c}\text { Culture } \\
\text { et } \\
\text { site }\end{array}$} & \multirow{2}{*}{\multicolumn{2}{|c|}{$\begin{array}{c}\text { Echantillon } \\
\mathrm{s}=\text { surface } \\
\mathrm{p}=\text { profondeur }\end{array}$}} & \multicolumn{2}{|c|}{ Matière organique } & \multirow{2}{*}{$\begin{array}{c}(\mathrm{A}+\mathrm{LF}) \% \\
0-20 \mu\end{array}$} & \multicolumn{2}{|c|}{ Phosphore (ppm P) } \\
\hline & & & & $\mathrm{C} \%$ & $\mathrm{C} / \mathrm{N}$ & & Total & Assimilable \\
\hline \multirow{10}{*}{ Ferrisol } & \multirow{7}{*}{$\begin{array}{c}\text { Canne } \\
(\text { (e Gallion ») }\end{array}$} & TN & $s$ & 42,6 & 9,9 & 60 & 288 & 9 \\
\hline & & $\mathrm{TC}$ & $\mathrm{s}$ & 29,6 & 11,5 & 67,0 & 537 & 13 \\
\hline & & & $\mathrm{p}$ & & & 65,9 & 240 & \\
\hline & & $\mathrm{R} 10$ & $s$ & 9,4 & 14,7 & 85,0 & 192 & 4 \\
\hline & & & $\mathrm{p}$ & 4,9 & 17,5 & 87 & 179 & 4 \\
\hline & & $\mathrm{R} 12$ & $s$ & 10,0 & 12,3 & 74,0 & 432 & 17 \\
\hline & & & $\mathrm{p}$ & 4,2 & & 75,5 & 371 & 13 \\
\hline & \multirow{3}{*}{$\begin{array}{c}\text { Canne } \\
\text { (« le Mauny ») }\end{array}$} & $\mathrm{TN}$ & $\mathrm{s}$ & 37,1 & 11,8 & 65,4 & 445 & 6 \\
\hline & & R6 & $\mathrm{s}$ & 12,6 & 13,4 & 77 & 371 & 4 \\
\hline & & & $\mathrm{p}$ & 4,7 & 18,8 & 73 & 371 & 6 \\
\hline \multirow{5}{*}{$\begin{array}{l}\text { Sol } \\
\text { brun rouille }\end{array}$} & \multirow{5}{*}{$\begin{array}{c}\text { Canne } \\
\text { (« Ste Marie ») }\end{array}$} & $\mathrm{TN}$ & $\mathrm{s}$ & 39,2 & 11,8 & 49,2 & 838 & 43 \\
\hline & & & $\mathrm{p}$ & & & 54,9 & & \\
\hline & & $\mathrm{TC}$ & $S$ & 19,0 & 11,2 & 69,0 & 812 & 65 \\
\hline & & & $\mathbf{p}$ & 5,0 & & 69,5 & 314 & 26 \\
\hline & & $\mathrm{R} 10$ & $\mathrm{~s}$ & 11,5 & 17,3 & & 511 & 22 \\
\hline \multirow{11}{*}{ Andosol } & \multirow{6}{*}{$\begin{array}{c}\text { Canne } \\
\text { («St Etienne ») }\end{array}$} & \multirow[t]{2}{*}{$\mathrm{TN}$} & $\mathrm{s}$ & 66,9 & 10,3 & 26,7 & 786 & 13 \\
\hline & & & $\mathrm{p}$ & 16,7 & 9,8 & 26,9 & 327 & 6 \\
\hline & & \multirow[t]{2}{*}{$\mathrm{TC}$} & $S$ & 32,7 & 13,5 & 42,1 & 742 & 22 \\
\hline & & & $\mathrm{p}$ & 26,5 & 8,3 & 37,6 & 450 & 9 \\
\hline & & \multirow[t]{2}{*}{ R8 } & $\mathrm{s}$ & 13,5 & 12,9 & 37,2 & 415 & 4 \\
\hline & & & $\mathrm{p}$ & 8,7 & 16,1 & 45,4 & 393 & traces \\
\hline & \multirow{5}{*}{$\begin{array}{c}\text { Banane } \\
(« \text { St Joseph ») }\end{array}$} & $\mathrm{TN}$ & $\mathrm{s}$ & 67,5 & 11,8 & 51,6 & 655 & 9 \\
\hline & & & $\mathrm{p}$ & & & 51,0 & 620 & traces \\
\hline & & $\mathrm{TC}$ & $\mathrm{s}$ & 48,9 & 10,3 & 30,7 & 786 & 17 \\
\hline & & & $\mathrm{p}$ & 42,5 & 9,6 & 16,0 & 567 & 2 \\
\hline & & R7 & s & 24,1 & 9,1 & & 371 & 9 \\
\hline
\end{tabular}

\section{RÉSULTATS ET DISCUSSION}

A. Effet de la profondeur sur la fixation du phosphore : comparaison des échantillons (s) et (p)

Les résultats sont groupés dans le tableau 3. Les valeurs de $r_{1} / R$, qui diminuent lorsque, pour un site donné, on passe de l'horizon de surface $(0-15 \mathrm{~cm})$ à l'horizon sous-jacent, indiquent que le pouvoir fixateur du sol des échantillons TN et TC, croît avec la profondeur, indépendamment du type pédologique. L'observation du tableau 3 autorise à émettre l'hypothèse qu'en un site déterminé, la variation de teneur en matière organique d'un horizon à l'autre suffit à expliquer leurs différences au regard de la fixation des ions phosphatés.

Ces résultats sur l'effet profondeur sont à rapprocher de ceux observés dans une étude antérieure (FARDEAU \& JAPPE, 1978) dont les conclusions soulignaient le danger des labours profonds, le mélange des horizons inférieurs (au-delà de $20 \mathrm{~cm}$ ) et superficiels entraînant une augmentation significative du pouvoir fixateur de l'horizon Ap. Ceci explique que les valeurs observées pour les échantillons $R$ soient indépendantes de la profondeur puisque les horizons actuels de surface sont soit d'anciens horizons B ou C dont les teneurs en matière organique n'ont pas encore atteint celles des traitements TC, soit encore des mélanges de l'ensemble des anciens horizons.

\section{B. Comparaison des échantillons de surface : effet des opérations de nivellement}

Tous les sols ont un pouvoir fixateur élevé à très élevé : $r_{1} / R$ est toujours inférieur à 0,2 (tabl. 3 ).

Cette conclusion, déduite de la donnée isotopique $\mathrm{r}_{1} / \mathrm{R}$, a été confirmée par l'analyse, selon les critères habituels, des isothermes de LANGMUIR qui permettent, théoriquement, de déterminer la quantité maximale de substrat qui est susceptible d'être adsorbée sur un support (TRUONG et al., 1974) : elle est comprise entre 400 et 2000 p.p.m. P selon les échantillons. Nous n'en présentons pas cependant les résultats détaillés tant les interprétations classiques semblent désormais discutables. En effet, non seulement l'hypothèse de base qui consiste à estimer que tous les ions retenus sont seulement adsorbés est inexacte, mais encore chacun peut constater, comme pour le potassium d'ailleurs (OUVRY, 1985), que le sol continue de retenir des ions phosphate au-delà de la valeur maximale d'adsorption déterminée par les isothermes de LANGMUIR. 
TABLEAU 3

Caractéristiques des cinétiques de dilution isotopique. Comparaison des échantillons de surface et de profondeur. Main characteristics of isotope dilution kinetics in 0-15 and $30-50 \mathrm{~cm}$ layers.

\begin{tabular}{|c|c|c|c|c|c|c|}
\hline \multirow{2}{*}{ Type de sol } & \multirow{2}{*}{ Culture } & \multirow{2}{*}{\multicolumn{2}{|c|}{ Echantillon $(*)$}} & \multicolumn{3}{|c|}{ Caractéristiques de la cinétique } \\
\hline & & & & n & $r_{1} / R$ & $\begin{array}{c}\mathrm{M}_{1} \\
\mathrm{ppmP}\end{array}$ \\
\hline \multirow{11}{*}{ Ferrisol } & \multirow{7}{*}{$\begin{array}{c}\text { Canne } \\
\text { " le Gallion » }\end{array}$} & TN & $s$ & 0,43 & 0,095 & 0,23 \\
\hline & & TC & $\mathrm{s}$ & 0,41 & 0,10 & 0,17 \\
\hline & & \multirow{3}{*}{$\mathrm{R} 10$} & $\mathrm{p}$ & 0,43 & 0,017 & 0,19 \\
\hline & & & $\mathrm{s}$ & 0,42 & 0,010 & 0,09 \\
\hline & & & $\mathrm{p}$ & 0,22 & 0,039 & 0,015 \\
\hline & & \multirow{2}{*}{$\mathrm{R} 12$} & $\mathrm{~s}$ & 0,27 & 0,015 & 0,14 \\
\hline & & & $\mathrm{p}$ & 0,19 & 0,010 & 0,11 \\
\hline & & TN & $\mathrm{s}$ & 0,37 & 0,070 & 0,21 \\
\hline & Canne & & $\mathrm{p}$ & 0,46 & 0,003 & 0,03 \\
\hline & « le Mauny » & R6 & $\mathrm{s}$ & 0,23 & 0,0064 & $<0,01$ \\
\hline & & & $\mathrm{p}$ & 0,15 & 0,0036 & 0,016 \\
\hline \multirow{5}{*}{$\begin{array}{c}\text { Sol } \\
\text { brun rouille }\end{array}$} & \multirow{5}{*}{$\begin{array}{c}\text { Canne } \\
\text { «Ste Marie » }\end{array}$} & \multirow[t]{2}{*}{ TN } & s & 0,40 & 0,038 & 0,41 \\
\hline & & & $\mathrm{p}$ & 0,35 & 0,015 & 0,09 \\
\hline & & \multirow[t]{2}{*}{$\mathrm{TC}$} & $\mathrm{s}$ & 0,25 & 0,027 & 0,17 \\
\hline & & & $\mathrm{p}$ & 0,19 & 0,0044 & $<0,01$ \\
\hline & & R10 & $\mathrm{s}$ & 0,26 & 0,025 & $<0,01$ \\
\hline \multirow{11}{*}{ Andosol } & \multirow{6}{*}{$\begin{array}{c}\text { Canne } \\
\text { « St Etienne » }\end{array}$} & \multirow[t]{2}{*}{ TN } & $\mathrm{s}$ & 0,50 & 0,023 & 0,08 \\
\hline & & & $\mathrm{p}$ & 0,50 & 0,032 & $<0,01$ \\
\hline & & \multirow[t]{2}{*}{ TC } & $\mathrm{s}$ & 0,33 & 0,018 & $<0,01$ \\
\hline & & & $\mathrm{p}$ & 0,37 & 0,0073 & 0,015 \\
\hline & & \multirow{2}{*}{$\mathrm{R} 8$} & $\mathrm{~s}$ & 0,26 & 0,012 & $<0,01$ \\
\hline & & & $\mathrm{p}$ & 0,24 & 0,002 & $<0,01$ \\
\hline & \multirow{5}{*}{$\begin{array}{c}\text { Banane } \\
\text { «St Joseph » }\end{array}$} & $\mathrm{TN}$ & $s$ & 0,50 & 0,070 & 0,74 \\
\hline & & \multirow{3}{*}{$\mathrm{TC}$} & $\mathrm{p}$ & 0,44 & 0,010 & 0,16 \\
\hline & & & $\mathrm{s}$ & 0,48 & 0,110 & 0,92 \\
\hline & & & $\mathrm{p}$ & 0,29 & 0,037 & 0,13 \\
\hline & & R7 & $\mathrm{s}$ & 0,25 & 0,042 & 0,25 \\
\hline
\end{tabular}

Tous les sols sont très pauvres en phosphore assimilable. En effet les valeurs de $\mathbf{M}_{1}$, qui sont les quantités de phosphore en solution, sont très faibles. Il en résulte que la quantité de phosphore isotopiquement échangée en $1 \mathrm{mn}$ (calculée à partir de la relation $\mathrm{E}_{1}=\frac{\mathrm{R}}{\mathrm{r}_{1}} \times \mathrm{M}_{1} \quad$ qui représente le phosphore le plus utilisable), est toujours très faible.

Pour un même type de sol représentatif d'un site donné, $M_{1}$ et $r_{1} / R$ diminuent lorsqu'on passe du traitement $\mathrm{TC}$ à $\mathrm{R}$ tandis que $\mathrm{n}$ augmente ou reste constant si sa valeur est déjà élevée pour TC. Ces résultats sont à rapprocher de ceux que l'on obtient au cours des études d'évolution de ces différents paramètres durant la phase d'appauvrissement d'un sol en phosphore assimilable (FARDEAU et al., 1977 ; FARDEAU, 1981).

Ainsi, cet ensemble de variations cohérentes des divers paramètres des cinétiques de dilution isotopique illustre à la fois la forte augmentation du pouvoir de fixation et l'appauvrissement de l'horizon de surface actuel en formes de phosphates mobiles à la suite des pratiques de remodelage. Ces modifications défavorables ont eu lieu malgré l'existence d'un $\mathrm{pH}$ égal ou supérieur à celui des sols sous culture beaucoup plus ancienne, ce qui aurait dû, tout au contraire, limiter l'adsorption et la fixation des ions phosphate sur les hydroxydes de fer et d'aluminium.
$\mathrm{Si}$, comme le laissait supposer la comparaison des résultats des horizons de surface et de profondeur, seul le paramètre quantité de matière organique intervenait, les pouvoirs fixateurs auraient dû se classer dans l'ordre $\mathrm{TN}<\mathrm{TC}<\mathrm{R}$, TN devant être très supérieur à $\mathrm{TC}$, les teneurs en matière organique variant d'un facteur voisin de 2 . La comparaison des horizons de surface possédant des teneurs en éléments fins et une minéralogie proches, c'est-à-dire pour les ferrisols $T N, T C, R_{10}$ et $R_{12}$, et, pour les andosols, $T C$ et $R_{8}$ ainsi que $T N$ et $R_{7}$, indique que leur pouvoir fixateur à l'égard des ions phosphate se classe dans l'ordre $\mathrm{TC}<\mathrm{TN}<\mathrm{R}$; ceci est vrai en particulier pour le ferrisol "le Gallion » où TC $<\mathrm{TN}$, malgré une teneur en éléments fins légèrement plus élevée et un pourcentage de carbone presque 2 fois plus faible. Enfin l'augmentation de 15 à 20 p. 100 de la teneur en éléments fins entre TN et TC, tant pour le sol brun rouille que pour l'andosol St-Etienne, ne provoque qu'une très légère augmentation du pouvoir fixateur (diminution de $r_{1} / R$ ), et ce, malgré des teneurs en matière organique totale également 2 fois plus basses. On peut donc penser qu'au regard des problèmes de fixation des ions phosphates par les sols, il existe un facteur qualité de la matière organique dans la mesure où celle originaire de la forêt est moins "efficace " que celle provenant de zones cultivées pour limiter la fixation. 
L'examen des valeurs de phosphore " assimilable » (tabl. 2) est en accord avec cette analyse dans la mesure où, quel que soit le sol, on a toujours TN $<$ TC.

D'autres études conduites en Afrique tropicale donnent la même tendance; il semblerait donc que les pratiques agricoles des siècles passés aient abouti à une augmentation de la disponibilité du phosphore pour la plante par rapport aux zones sous forêts.

\section{Effets des apports de phosphates solubles dans les horizons de surface $T C$ et $R$ : fixation des ions phosphates}

Des travaux antérieurs (FARDEAU \& JAPPE, 1980) indiquent qu'il est illusoire d'espérer modifier par des fumures de redressement le phosphore assimilable de tels sols. Cet aspect est analysé de nouveau sur quelques-uns des échantillons.

Seuls les échantillons prélevés dans l'horizon de surface ont été traités. Les résultats sont groupés dans le tableau 4.

Les apports de $66 \mu \mathrm{gP} . \mathrm{g}^{-1}$ sol qui représentent des fertilisations d'environ $450 \mathrm{~kg} \mathrm{P}_{2} \mathrm{O}_{5} \cdot \mathrm{ha}^{-1}$ ne permettent pas de dépasser, dans le cas le plus favorable, des quantités de phosphore en solution supérieures à $3,25 \mu \mathrm{gP} . \mathrm{g}^{-1}$ sol, ce qui ne représente que $4 \mathrm{p} .100 \mathrm{de}$ l'ajout. Il faut noter que, dans cette expérimentation, les phénomènes d'alternance d'humectation et de dessiccation toujours présents au niveau du champ n'ont pas été pris en compte : ils auraient encore accru la fixation du phosphore (BLANCHET, 1959).

Même à l'issue d'un apport de phosphate soluble important, les valeurs de $r_{1} / R$, à une exception près, sont inférieures à 0,2 : ces sols restent classés dans la catégorie des sols à fort pouvoir fixateur (FARDEAU \& JAPPE, 1978).
Une fertilisation de redressement ne permet pas d'atteindre pour un sol les caractéristiques d'un autre, si ceux-ci diffèrent initialement de façon siğnificative par leur pouvoir fixateur. C'est ce qu'illustre la figure 1 où, dans les exemples traités, aucune des

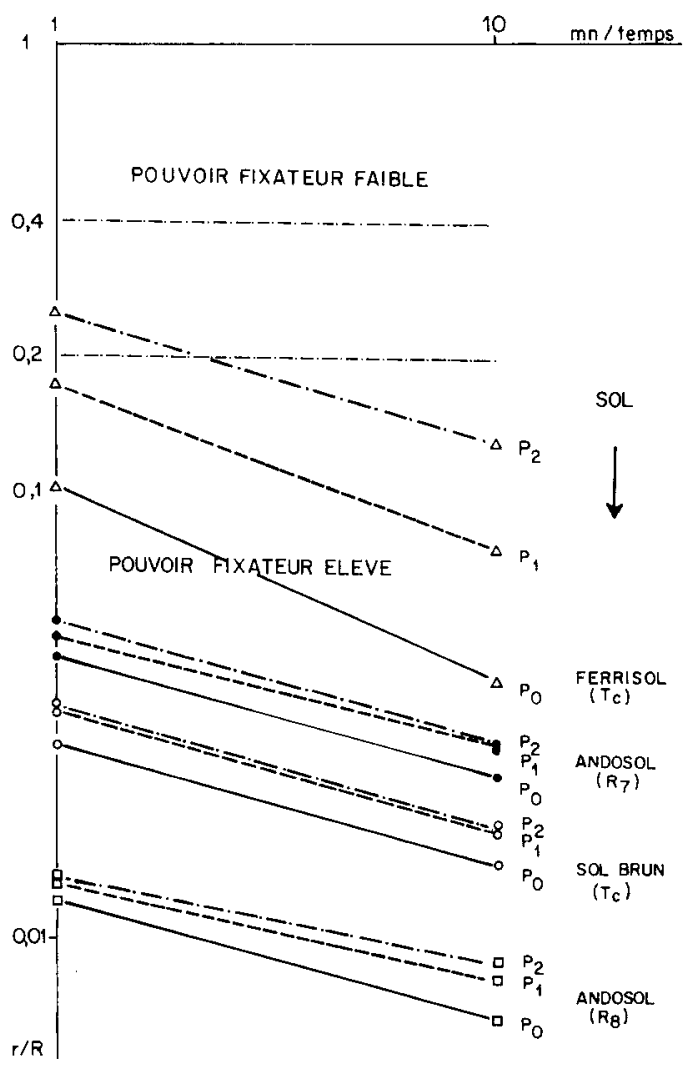

Figure 1

Cinétique de dilution isotopique dans divers types de sols. Isotope dilution kinetics for different soil types.

TABLEAU 4

Effet d'apports de phosphates solubles sur les indices des cinétiques de dilution isotopique. Effect of soluble phosphate fertilizers on the main characteristics of isotope dilution kinetics.

\begin{tabular}{|c|c|c|c|c|c|c|c|c|c|c|c|}
\hline \multirow{3}{*}{$\begin{array}{l}\text { Type de sol } \\
\text { et culture }\end{array}$} & \multirow{3}{*}{$\begin{array}{l}\text { Doses } \mathbf{P} \\
\text { apporté } \\
\text { ppmP }\end{array}$} & \multicolumn{10}{|c|}{ Traitements } \\
\hline & & \multicolumn{5}{|c|}{ Témoin cultivé } & \multicolumn{5}{|c|}{ Sols remodelés } \\
\hline & & \multicolumn{2}{|c|}{ éch. } & \multirow{7}{*}{$\begin{array}{c}\mathbf{n} \\
0,41 \\
0,35 \\
0,29\end{array}$} & \multirow{7}{*}{$\begin{array}{l}r_{1} / R \\
0,10 \\
0,17 \\
0,25\end{array}$} & \multirow{7}{*}{\begin{tabular}{r}
\multicolumn{1}{c}{$\begin{array}{c}\mathrm{M}_{1} \\
\text { ppmP }\end{array}$} \\
0,17 \\
1,17 \\
3,25
\end{tabular}} & \multicolumn{2}{|c|}{ éch. } & \multirow{2}{*}{$\begin{array}{c}\mathrm{n} \\
0,42\end{array}$} & \multirow{2}{*}{$\frac{r_{1} / R}{0,010}$} & \multirow{2}{*}{$\frac{\begin{array}{c}\mathrm{M}_{1} \\
\mathrm{ppmP}\end{array}}{<0,01}$} \\
\hline Ferrisol & 0 & $\mathrm{TC}$ & s & & & & $\mathrm{R} 10$ & $s$ & & & \\
\hline Canne & 33 & & & & & & & & 0,27 & 0,013 & 0,03 \\
\hline & 66 & & & & & & & & 0,27 & 0,015 & 0,04 \\
\hline & 0 & & & & & & $\mathrm{R} 12$ & s & 0,27 & 0,015 & $<0,01$ \\
\hline & 33 & & & & & & & & 0,28 & 0,023 & 0,08 \\
\hline & 66 & & & & & & & & 0,28 & 0,025 & 0,08 \\
\hline Sol & 0 & $\mathrm{TC}$ & s & 0,25 & 0,027 & 0,17 & R10 & $\mathrm{s}$ & 0,26 & 0,025 & $<0,01$ \\
\hline brun rouille & 33 & & & 0,25 & 0,032 & 0 & & & 0,21 & 0,029 & 0,08 \\
\hline Canne & 66 & & & 0,25 & 0,033 & 0,50 & & & 0,18 & 0,039 & 0,25 \\
\hline Andosol & 0 & $\mathrm{TC}$ & s & 0,33 & 0,018 & $<0,01$ & $\mathrm{R} 8$ & s & 0,26 & 0,012 & $<0,01$ \\
\hline Canne & 33 & & & 0,19 & 0,020 & $<0,01$ & & & 0,20 & 0,013 & $<0,01$ \\
\hline & 66 & & & 0,20 & 0,023 & 0,02 & & & 0,18 & 0,014 & 0,33 \\
\hline Banane & 0 & $\mathrm{TC}$ & $\mathrm{s}$ & 0,48 & 0,110 & 0,92 & $\mathrm{R} 7$ & $s$ & 0,25 & 0,042 & 0,25 \\
\hline & 33 & & & 0,42 & 0,120 & 1,25 & & & 0,24 & 0,047 & 0,33 \\
\hline & 66 & & & 0,43 & 0,150 & 1,58 & & & 0,27 & 0,052 & 0,58 \\
\hline
\end{tabular}


droites représentatives des cinétiques de dilution isotopique des échantillons ayant reçu la dose $\mathrm{P}_{2}$ ne chevauche celle de l'échantillon $P_{0}$ situé immédiatement après.

On note également, par comparaison des échantillons Ferrisol TC à Ferrisol $\mathrm{R}_{10}$ ou Andosol $\mathrm{R}_{8}$, que les variations relatives de $r_{1} / R$ et de $M_{1}$, à l'issue d'un ajout de phosphate, sont d'autant plus grandes que $\mathrm{r}_{1} / \mathrm{R}$ du sol témoin est plus élevé. Il en résulte qu'une modification positive du pool de phosphate assimilable par des fertilisations à base de formes soluble-eau d'engrais phosphatés est d'autant plus aisée que le pouvoir fixateur initial est plus faible, c'est-à-dire que $r_{1} / R$ est plus élevé.

Différents chercheurs ont signalé que de fortes fertilisations en sol pauvre ne permettaient pas d'atteindre des niveaux de rendements aussi élevés qu'en sol riche (BARBIER \& TROCMÉ, 1964) ; nous pensons que nos observations peuvent, pour partie, expliquer cet effet « vieille graisse » des sols.

Enfin, la figure 2 illustre une des conséquences du remodelage sur 2 types de sol (ferrisol et andosol) ayant reçu diverses doses de phosphate. Le remodelage transforme le ferrisol à pouvoir fixateur "moyen » en un sol à fort pouvoir fixateur pour lequel l'accroissement des réserves mobiles ne peut en aucun cas être obtenu par des apports, même massifs, d'engrais phosphatés.

\section{Comment aborder la fertilisation phosphatée de ces sols ?}

Ces sols sont pauvres à très pauvres en phosphore assimilable et à fort pouvoir fixateur. Après les apports de phosphates solubles (tabl. 4) de

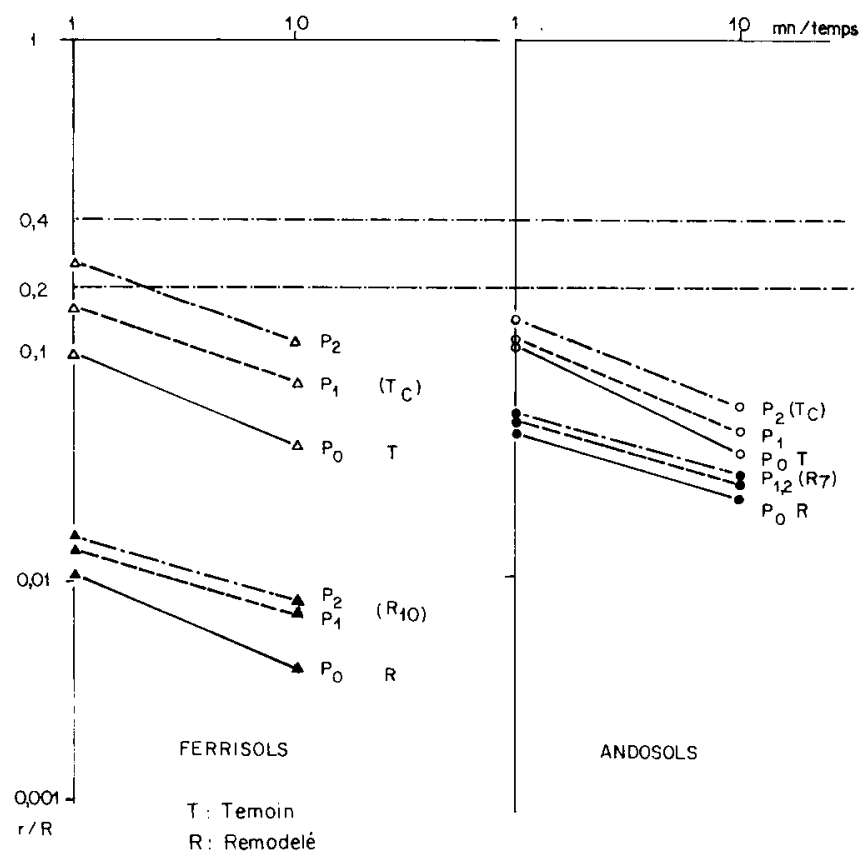

Figure 2

Cinétique de dilution isotopique de divers échantillons fertilisés. Comparaison surface-profondeur.

Isotope dilution kinetics of different fertilized samples. Soil-subsoil comparison.
66 p.p.m. P, qui correspondent à une fumure voisine de $450 \mathrm{~kg} \mathrm{P}_{2} \mathrm{O}_{5} \mathrm{ha}^{-1}$, la concentration en phosphore de la solution du sol peut être divisée par rapport à sa valeur initiale, 6,6 p.p.m. P, d'un facteur $1600=$ $6,6 / 0,004$ pour le cas le plus défavorable ( $\left(\mathrm{sol} R_{10} \mathrm{~s}\right.$ ), et au moins d'un facteur 100 dans les autres sols. De plus, les ions phosphatés retenus par le sol ne se maintiennent que très peu sous forme isotopiquement échangeable puisque, par rapport aux sols témoins $\left(\mathrm{P}_{0}\right)$, les cinétiques ne sont que très peu modifiées par les apports. Dans ces conditions, l'action d'un engrais phosphaté sera limitée à ses effets immédiats sur la culture.

C'est pourquoi on se doit de :

1) Comparer différentes formes d'engrais : «solubles » ou « insolubles ». On sait que si le $\mathrm{pH}$ du sol est supérieur à 6,2 , les formes insolubles ont des coefficients d'utilisation par les récoltes inférieurs à 1 p. 100 (FARDEAU et al., 1983 ; JADIN et al., 1983). Par contre, si le pH est inférieur à 6,2, le coefficient d'utilisation du phosphore des formes insolubles tels que des phosphates naturels calciques peut être aussi élevé que celui obtenu avec une forme soluble notamment en choisissant leur origine géographique et leur finesse de mouture (TRUONG, 1984).

Ces observations ont été faites pour des sols des régions tropicales (ROCHE et al., 1968) ou tempérées (JAHIEL \& MOREL, 1983). C'est pourquoi nous pensons que dans les sols acides étudiés ici, des phosphates naturels pourraient être utilisés en fumures annuelles (AYRES \& HAGIHARA, 1961 ; GACHON, 1977) ; leur efficacité serait probablement égale à celle des engrais solubles.

2) Rechercher, pour une forme d'engrais donné, les techniques culturales conduisant à l'efficacité immédiate maximale de l'engrais: fractionnement de l'apport, localisation, association avec des amendements organiques... En ce qui concerne ce dernier point, les mécanismes d'interactions phosphore de l'engrais/matière organique sont actuellement mal cernés; quelques résultats de cette étude militent en faveur d'un effet positif de la matière organique. Certes, l'hypothèse contraire pourrait dans un premier temps être émise avec les résultats obtenus pour les échantillons riches en matière organique des traitements «végétation naturelle » mais, en réalité, c'est un critère «qualité de la matière organique » qui apparaît.

\section{CONCLUSION}

Appliquée à l'étude d'échantillons de divers types de sols de la Martinique, la technique de cinétique de dilution isotopique des ions phosphate dans les systèmes sol-solution en état stationnaire permet de préciser le pouvoir fixateur de ces sols, l'effet du remodelage des terrains et les conséquences qui en résultent en matière de fertilisation phosphatée.

- Le pouvoir fixateur des sols examinés est nettement plus élevé en moyenne que celui des sols de la zone tempérée.

- La comparaison des horizons humifères $\mathrm{A}$ et des horizons sous-jacents $\mathrm{B}$ ou $\mathrm{C}$ à minéralogies identi- 
ques fait apparaître le rôle favorable d'un certain type de matière organique sur l'abaissement du pouvoir fixateur : celle que l'on rencontre dans les sols cultivés de longue date.

- Les conséquences des opérations de génie civil de remodelage des sols destinées à favoriser la mécanisation des cultures sont particulièrement néfastes en ce qui concerne la mobilité des ions phosphates des sols.
- Compte tenu de l'acidité de ces sols et du niveau de leur pouvoir fixateur, il semble judicieux de conseiller une fertilisation phosphatée à base de phosphates naturels.

Reçu le 5 décembre 1984 Accepté le 23 septembre 1985.

\section{RÉFÉRENCES BIBLIOGRAPHIQUES}

Ayres A. S., Hagihara H. H., 1961. Effectiveness of raw rock phosphate for sugar cane. Soil Sci., 91 (6), 383-387.

Barbier G., Trocmé S., 1964. Rôle comparé des engrais et des réserves nutritives du sol dans la production des récoltes. $Z$. Pflanzenernahr., 109 (2), 113-120.

Barbier G., Tyszkiewicz E., 1952. Mobilité des ions phosphoriques fixés dans le sol, étudiée au moyen de ${ }^{32} \mathrm{P}$. Assoc. Intern. Sci. Sol, Commissions II et IV, Dublin, 79-81.

Blanchet R., 1959. Energie d'adsorption des ions minéraux par les colloïdes du sol et nutrition minérale des plantes. Ann. agron., 10, 5-52, 125-153 et 267-307.

Chevignard T., 1982. Etude des sols remodelés de la Martinique. Rapp. Ronéo, ORSTOM, Fort-de-France, 29 p.

Colmet-Daage F., Lagache P., 1965. Caractéristiques de quelques groupes de sols dérivés de roches volcaniques aux Antilles françaises. Cah. ORSTOM, Série Pédologie, 3 (2), 91-122.

Colmet-Daage F., Gautheyrou M., Kimpe C., Fusil G., 1972. Dispersion et étude des fractions fines de sols à allophane des Antilles et d'Amérique latine. $1^{\text {re }}$ partie. Dispersion. Cah. ORSTOM, Série Pédologie, 10 (2), 169-192.

Demolon A., Boischot P., Lajon J., 1953. Réaction des divers sols à l'apport des engrais phosphatés solubles, son importance dans l'établissement des fumures. Ann. agron. 4, 291-330.

Fardeau J. C., 1981. Cinétique de dilution isotopique et phosphore assimilable des sols. Thèse de Doctorat, Université de Paris VI, 192 p.

Fardeau J. C., Jappe J., 1978. Analyse par dilution isotopique de la fertilité et de la fertilisation phosphorique de quelques sols du Québec. Can. J. Soil Sci. 58, 251-258.

Fardeau J. C., Jappe J., 1980. Choix de la fertilisation phosphorique des sols tropicaux : emploi du phosphore 32. Agron. trop., 35 (3), 225-231.

Fardeau J. C., Jappe J., 1982. Intérêt des cinétiques d'échanges isotopiques pour la caractérisation du phosphore assimilable des sols. Sci. Sol, Bull. Assoc. fr. Etud. Sol, 2, 113-124.

Fardeau J. C., Chabouis C., Hetier J. M., 1977. Méthode des cinétiques de dilution isotopique des ions $\mathrm{PO}_{4}$ et $\mathrm{K}$ : application à l'analyse des modifications de fertilité dans les essais de très longue durée. Sci. Sol, Bull. Assoc. fr. Etude Sol, 2, 111-122.

Fardeau J. C., Migadel F., Gjermani A., Malja S., 1983. Efficacité d'un phosphate à faible teneur dans quelques sols d'Albanie. Mesu- res au moyen de ${ }^{32} \mathrm{P} .3^{e}$ congrès int. sur les composés phosphorés, 4 6 octobre 1983, Bruxelles, 519-532.

Fardeau J. C., Morel C., Jappe J., 1985. Cinétique d'échange des ions phosphate dans les systèmes sol-solution. Vérification expérimentale de l'équation théorique. C. R. Acad. Sci. Paris 300, Série 3 (8), 371-375.

Fox R. L., Kamprath E. J., 1970. Phosphate sorption isotherms for evaluating the phosphate requirements of soils. Soil Sci. Soc. Am. Proc., 34, 902-907.

Gachon L., 1969. Les méthodes d'appréciation de la fertilité phosphorique des sols. Bull. Assoc. fr. Etud. Sol, 17-29.

Gachon L., 1977. Utilité d'un bon niveau de réserves phosphatées du sol. Phosphore Agric., 70, 27-33.

Jadin P., Fardeau J. C., Jappe J., 1983. Correction de la carence phosphorique des sols (ferral-sols) sous cacaoyers (Theobroma cacao). $3^{e}$ Congrès int. sur les composés phosphorés, 4-6 oct. 1983, Bruxelles, 327-344.

Jahiel M., Morel C., 1983. Analyse du devenir de différents phosphates dans plusieurs sols de pH variant de 5,2 à 6,6. Mémoire de fin d'études, I.S.A. Lille, 119 pages.

John M. K., 1970. Colorimetric determination of phosphorus in soil and plant material with ascorbic acid. Soil Sci., 109 (4), 214-222.

Marini P., 1965. Contribution à l'étude de la rétention des anions phosphoriques par les argiles. Thèse Doct. Ing., Toulouse, $112 \mathrm{p}$.

Ouvry J. F., 1985. Etude expérimentale de la dynamique du potassium sur deux sols tropicaux. Thèse Doct. Ing., Montpellier, $250 \mathrm{p}$

Roche P., Velly J., N. G. O. Chan Bang, 1968. Comparaison de diverses natures d'engrais azotés et d'engrais phosphatés en rizière. Coll. sur la fertilité des sols tropicaux, Tananarive 19-25 nov. 1968, I.R.A.T. édit., 1122-1126.

Sheldon R., 1982. Les phosphates. Pour la Science, août 1982, 34 67.

Truong B., Bertrand R., Burdin S., Pichot J., 1974. Contribution à l'étude du phosphore dans les sols dérivés de roches volcaniques de l'Ile de la Réunion (Mascareignes). Actions du carbonate et du silicate de calcium. Agron. trop., 29 (6-7), 1-12.

Truong B., 1984. Contraintes à l'utilisation des phosphates naturels. Mise au point d'engrais alternatifs. Séminaire sur «Les contraintes a. urllisution des engrais ", Yamoussoukro, 11-14 déc. 1984. 